\title{
First extraction of Transversity from data on lepton-hadron scattering and hadronic collisions
}

\author{
Marco Radici* \\ INFN Sezione di Pavia, via Bassi 6, I-27100 Pavia, Italy \\ E-mail: marco.radiciapv.infn.it
}

\begin{abstract}
We describe the extraction of the transversity distribution for the first time based on the global analysis of pion-pair production in deep-inelastic scattering and in proton-proton collisions with one transversely polarized proton. The extraction involves di-hadron fragmentation functions, which are taken from the analysis of the corresponding pion-pair production in electron-positron annihilation. We discuss the impact of COMPASS pseudodata from a future measurement of deep-inelastic scattering on transversely polarized deuterons. The knowledge of transversity is important among other things for detecting possible signals of new physics in high-precision lowenergy experiments.
\end{abstract}

23rd International Spin Physics Symposium - SPIN2018 -

10-14 September, 2018

Ferrara, Italy

\footnotetext{
* Speaker.
} 


\section{Introduction}

The transversely polarized parton distribution $h_{1}$ (transversity) is the least known of parton distribution functions (PDFs) because it is not diagonal in the helicity basis (in jargon, it is a chiral-odd function). As such, $h_{1}$ is suppressed in simple processes like inclusive deep-inelastic scattering (DIS). It can be measured only in processes with at least two hadrons: semi-inclusive DIS (SIDIS) or hadronic collisions. On the other hand, transversity is interesting because its first Mellin moment, the so-called tensor charge, can be linked to effects from physics beyond the Standard Model (BSM) [1].

We access transversity at leading twist and in the standard framework of collinear factorization. We consider the semi-inclusive production of two hadrons with small invariant mass, where there is a correlation between the transverse polarization of the quark directly fragmenting into the two hadrons and their transverse relative momentum [2]. In this case, the di-hadron SIDIS cross section (once integrated over partonic transverse momenta) contains a specific modulation in the azimuthal orientation of the plane containing the momenta of the two hadrons. The coefficient of this modulation is the simple product $h_{1} H_{1}^{\varangle}$ where $H_{1}^{\varangle}$ is a chiral-odd di-hadron fragmentation function (DiFF) quantifying the above correlation $[3,4,5]$. The function $H_{1}^{\varangle}$ can be independently determined by looking at correlations between the azimuthal orientations of two hadron pairs in back-to-back jets in $e^{+} e^{-}$annihilation $[6,7,8,9]$. The advantage of this method is that collinear factorization makes it possible to isolate the same combination $h_{1} H_{1}^{\varangle}$ also in proton-proton collisions [10], giving rise to an azimuthally asymmetric distribution of the final hadron pair when one of the two initial protons is transversely polarized [11].

Experimental data for the SIDIS asymmetry in the azimuthal distribution of final $\left(\pi^{+} \pi^{-}\right)$ pairs were collected by the HERMES collaboration for a proton target [12], and by the COMPASS collaboration for both protons and deuterons $[13,14]$. The azimuthal asymmetry in the distribution of back-to-back $\left(\pi^{+} \pi^{-}\right)$pairs in $e^{+} e^{-}$annihilation was measured by the BELLE collaboration [15], opening the way to the first parametrization of $H_{1}^{\varangle}$ [8]. In turn, this result was used in combination with the SIDIS data to extract the valence components of $h_{1}[16,17,18]$. Recently, the STAR collaboration released the first results for the predicted asymmetry in the azimuthal distribution of $\left(\pi^{+} \pi^{-}\right)$pairs produced in proton-proton collisions with a transversely polarized proton [19]. Here, we describe the extraction of the transversity PDF $h_{1}$ from a global fit of all these data for the semiinclusive production of $\left(\pi^{+} \pi^{-}\right)$pairs. The final results have been published in Ref. [20]. We also discuss the impact of COMPASS pseudodata from a future SIDIS measurement on deuterons [21].

\section{Formalism}

The relevant observables for our global fit are the following.

For SIDIS on a transversely polarized hadron, at leading twist the cross section contains the azimuthally asymmetric term $\sin \left(\phi_{R}+\phi_{S}\right) h_{1} H_{1}^{\varangle}$, where $\phi_{R}$ and $\phi_{S}$ are the azimuthal angles with respect to the scattering plane of the pair relative momentum and target polarization vector, respectively. The corresponding single-spin asymmetry is described in detail in Refs. [17, 18].

The $H_{1}^{\varangle}$ can be extracted from electron-positron annihilations leading to two correlated hadron pairs in opposite hemispheres. Again at leading twist, the cross section contains the azimuthally asymmetric term $\cos \left(\phi_{R}+\bar{\phi}_{R}\right) H_{1}^{\varangle} \bar{H}_{1}^{\varangle}$, where the barred quantities refer to the corresponding anti- 
quark emerging from the $e^{+} e^{-}$annihilation. From this, we can construct the so-called Artru-Collins asymmetry $[6,8]$ and extract the $H_{1}^{\varangle}$ by summing upon all pairs in one hemisphere, involving the number density of fragmenting (un)polarized quarks [8].

Lastly, for proton-proton collisions with one transversely polarized proton the cross section at leading order in the hard scale contains the azimuthally asymmetric term $\sin \left(\phi_{S}-\phi_{R}\right) f_{1}^{a} \otimes h_{1}^{b} \otimes$ $d \sigma_{a b^{\uparrow} \rightarrow c^{\uparrow} d} \otimes H_{1}^{\varangle c}$. The angles $\phi_{S}, \phi_{R}$ have the same meaning as before but with respect to the reaction plane of the collision process, the $d \sigma$ is the elementary cross section for the annihilation of a parton $a$ with a transversely polarized parton $b$ into the transversely polarized parton $c$ and the parton $d$ (summing over all undetected $d$ ). This term leads to the spin asymmetry described in Ref. [10]. Despite working in the collinear framework, the elementary combination $h_{1} H_{1}^{\varangle}$ happens convoluted with other ingredients in the numerator of such asymmetry, making its repeated computation during the fit very demanding. In order to speed up the execution of the fitting code, the well known workaround is to rewrite the parametric part of the integrand (in our case, the transversity) in terms of its Mellin anti-transform, such that most of the integrals can be computed before the minimization. In order to exploit this workaround, it is crucial that the Mellin transform of $h_{1}$ can be analytically calculated at any scale. To this purpose, we have chosen the following functional form for the valence component $q_{v}$ at the starting scale $Q_{0}^{2}=1 \mathrm{GeV}^{2}$ :

$$
\begin{aligned}
x h_{1}^{q_{v}}\left(x, Q_{0}^{2}\right) & =F^{q}(x) F_{\mathrm{SB}}^{q}(x), \\
F_{\mathrm{SB}}^{q}(x) & =N_{\mathrm{SB}}^{q} x^{a_{q}}(1-x)^{b_{q}}\left(1+c_{q} \sqrt{x}+d_{q} x+e_{q} x^{2}+f_{q} x^{3}\right), \\
F^{q}(x) & =N_{F}^{q} \frac{\mathscr{F}^{q}(x)}{\max _{x}\left[\left|\mathscr{F}^{q}(x)\right|\right]}, \quad \mathscr{F}^{q}(x)=x^{A_{q}}\left[1+B_{q} T_{1}(x)+C_{q} T_{2}(x)+D_{q} T_{3}(x)\right] .
\end{aligned}
$$

The $F_{\mathrm{SB}}$ in Eq. (2.2) is a fit to the Soffer bound at $Q_{0}^{2}$, whose analytic expression is listed in the appendix of Ref. [17]. Using the parameters listed in Tab.I of Ref. [20], the accuracy is of order $1 \%$ in the range $0.001 \leq x \leq 1$. In Eq. (2.3), the $T_{n}(x)$ are the Cebyshev polynomials of order $n$. The $N_{F}^{q}, A_{q}, B_{q}, C_{q}, D_{q}$, are the fitting parameters. For the moment, only the valence components of transversity can be accessed [17]; hence, we have in total 10 free parameters. If we impose the constraint $\left|N_{F}^{q}\right| \leq 1$, then $\left|F^{q}(x)\right| \leq 1$ for all $x$, and the $h_{1}^{q_{v}}$ in Eq. (2.1) automatically satisfies the Soffer inequality at any scale. Another constrain comes from the low- $x$ behavior of transversity, which from the above equations looks like $x h_{1}^{q_{v}}(x) \approx x^{A_{q}+a_{q}}$. For the given $a_{q}$, the parameter $A_{q}$ is strongly constrained by requiring that the tensor charge $\delta q\left(Q^{2}\right)$ is finite. We numerically evaluate the Mellin moment in the range $\left[10^{-6}, 1\right]$. In order to avoid uncontrolled extrapolation errors at $0 \leq x<10^{-6}$, we impose the condition $A_{q}+a_{q}>1 / 3$, which also grants that $\delta q$ is evaluated at $1 \%$ accuracy.

The statistical uncertainty of the global fit is studied at the $90 \%$ confidence level using the same bootstrap method as in our previous fits $[17,18]$. In the analysis of di-hadron $e^{+} e^{-}$data, the unconstrained gluon channel is assumed $D_{1}^{g}\left(Q_{0}^{2}\right)=0$. We parametrize this error by computing the asymmetry also with $\left.D_{1}^{g} Q_{0}^{2}\right)=D_{1}^{u}\left(Q_{0}^{2}\right) / 4$, or $D_{1}^{u}\left(Q_{0}^{2}\right)$. We have verified that these choices alter the $\chi^{2}$ of the $e^{+} e^{-}$fit in Ref. [8] by 10-50\%, keeping always $\chi^{2} /$ d.o.f. $\lesssim 2$. The number $M$ of replicas is fixed by reproducing the mean and standard deviation of the original data points. For each option, it turns out that 200 replicas are sufficient. Hence, we have in total $M=600$ replicas. 


\section{Results}
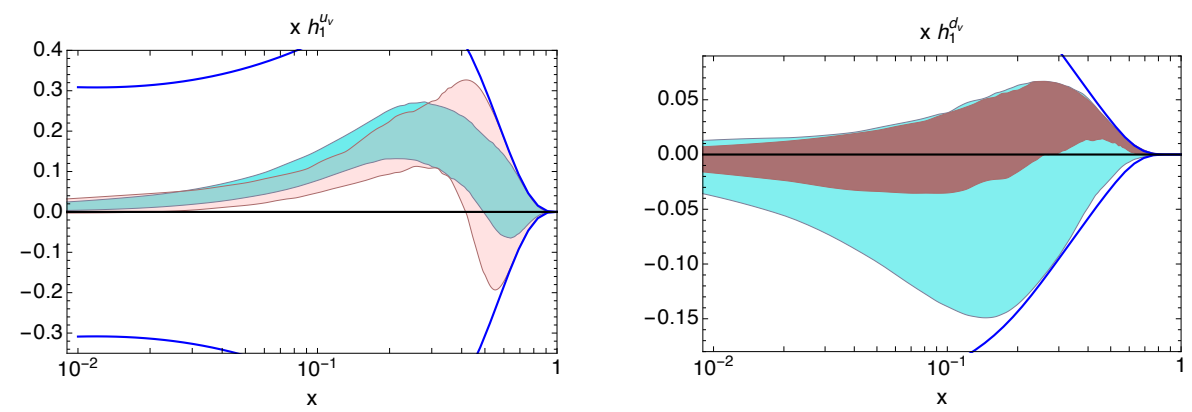

Figure 1: The transversity $x h_{1}$ as a function of $x$ at $Q^{2}=2.4 \mathrm{GeV}^{2}$. Blue lines represent the Soffer bounds. Cyan bands for the global fit of this work including all options $D_{1}^{g}\left(Q_{0}^{2}\right)=0, D_{1}^{u}\left(Q_{0}^{2}\right) / 4$ and $D_{1}^{u}\left(Q_{0}^{2}\right)$. Left panel for valence up quark: comparison with our previous fit in Ref. [18] (pink band). Right panel for valence down quark: comparison with this global fit with only $D_{1}^{g}\left(Q_{0}^{2}\right)=0$ (darker pink band in the foreground).

In Fig. 1, the transversity $x h_{1}$ is displayed as a function of $x$ at $Q^{2}=2.4 \mathrm{GeV}^{2}$. The blue lines represent the Soffer bounds. The left panel refers to the valence up component. Here, the pink band corresponds to our previous fit with only SIDIS and $e^{+} e^{-}$data [18]. The cyan band is the new global fit, including all options $D_{1}^{g}\left(Q_{0}^{2}\right)=0, D_{1}^{g}\left(Q_{0}^{2}\right)=D_{1}^{u}\left(Q_{0}^{2}\right) / 4$ and $D_{1}^{g}\left(Q_{0}^{2}\right)=D_{1}^{u}\left(Q_{0}^{2}\right)$ [20]. It turns out that this result is insensitive to the various choices for $D_{1}^{g}\left(Q_{0}^{2}\right)$ [20]. The uncertainty of our previous fit in Ref. [18] (the pink band) is comparable to the one obtained from the analysis of the Collins effect $[22,23]$. Hence, this plot indirectly illustrates how the result of our global fit compares with other independent methods of extracting transversity.

The right panel of Fig. 1 refers to the valence down component. Again, the cyan band corresponds to the global fit including all options for $D_{1}^{g}\left(Q_{0}^{2}\right)$. The darker pink band in the foreground shows how the result is modified by including only the option $D_{1}^{g}\left(Q_{0}^{2}\right)=0$. Evidently, the valence down component $h_{1}^{d_{v}}$ is very sensitive to $D_{1}^{g}$, in particular through the cross section for $p$ - $p$ collisions. This can be understood by considering that in the $p-p$ cross section the up and down contributions have equal weight and gluon effects are in principle active already at leading order, while in SIDIS the up counts eight times more than the down and the gluon effects appear only at next-to-leading order. For the very same reason, the deuteron is more sensitive to the down contribution than the proton, and better data on this target would help in reducing the uncertainty displayed in the right panel of Fig. 1. Data on $\left(\pi^{+} \pi^{-}\right)$multiplicities in $p$ - $p$ collisions would be very useful as well. Finally, we notice that the unnatural behavior of $h_{1}^{d_{v}}$ at $x \gtrsim 0.1$ obtained in Ref. [18] has disappeared. Hence, the STAR data have a significant impact on our knowledge of transversity.

In Fig. 2, we show the tensor charge $\delta q$ at $Q^{2}=4 \mathrm{GeV}^{2}$ and $90 \%$ confidence level. The upper panels refer to the up quark $\delta u$ (left) and to the down quark $\delta d$ (right); the lower panel to the isovector tensor charge $g_{T}=\delta u-\delta d$. In all panels, the blue point with label 2 is the result of our global fit [20], the red point with label 3 is the result of the phenomenological extraction based on the Collins effect in the transverse-momentum dependent framework [22], the magenta point with label 4 is also based on the Collins effect but in an extended parton model framework [23], the green point with label 5 is the result obtained with a nested Monte Carlo iterative approach to the Collins effect but with the additional constraint to reproduce the lattice results for $g_{T}$ [24], 


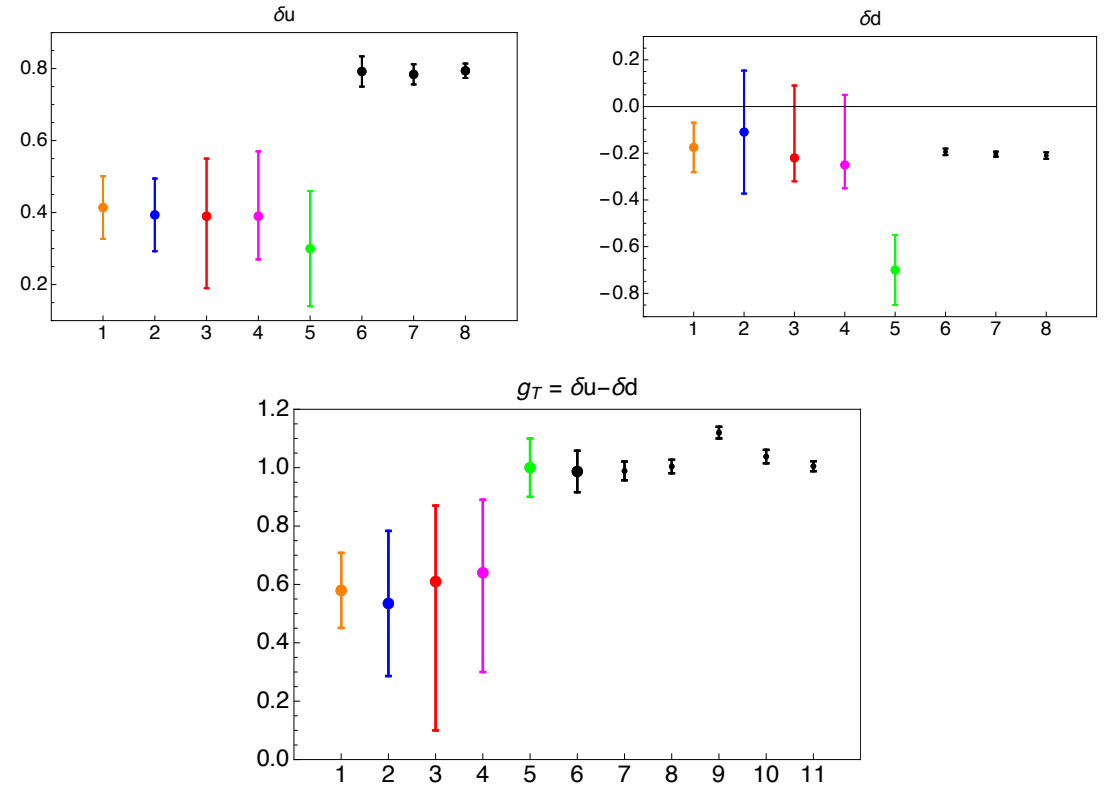

Figure 2: Upper panels: the tensor charges $\delta u$ (left) and $\delta d$ (right) at $Q^{2}=4 \mathrm{GeV}^{2}$ and $90 \%$ confidence level; orange point (label 1) for our global fit constrained by COMPASS deuteron pseudodata from Ref. [21], blue point (label 2) for our global fit from Ref. [20], red point (label 3) from Ref. [22], magenta point (label 4) from Ref. [23], green point (label 5) from Ref. [24], black points (labels 6, 7 and 8) from Refs. [25, 26, 27], respectively. Lower panel: the isovector tensor charge $g_{T}=\delta u-\delta d$ with same notations and color codes; additional lattice calculations in black points (labels 9, 10 and 11) from Refs. [28, 29, 30], respectively.

the black points with labels 6,7 and 8 are the latest lattice results from the PNDME $[25,26]$ and ETMC [27] collaborations, respectively. The black points in the lower panel with labels 9, 10 and 11 are the lattice results for $g_{T}$ from Refs. [28, 29, 30], respectively. Our results seem in very good agreement with other phenomenological extractions except for Ref. [24] on $\delta d$ and $g_{T}$. In general, there seems to be compatibility between lattice simulations and phenomenology only for $\delta d$.

In all panels of Fig. 2, the orange point with label 1 is the result of our global fit when including also the COMPASS pseudodata from a future SIDIS measurement on (transversely polarized) deuterons [21]. The bins are the same as in the older measurement [13] but the statistic is much higher and the error bars are approximately $60 \%$ of the ones from the more recent and precise measurement on a proton target [14]. For each bin, the central value of pseudodata is represented by the average value of all replicas that our global fit produces without using the pseudodata themselves. Since this choice is arbitrary, the orange points in Fig. 2 should be considered only to estimate how much the error bars shrink with respect to the blue points, that represent the result of our global fit when excluding these pseudodata. Indeed, we can notice that there is a significant reduction of the error for $\delta d$ (and, consequently, for $g_{T}$ ), as expected by following previous arguments on the quark content of deuteron targets. Still, the general incompatible trends of results from lattice simulation and phenomenology seem to be confirmed. In particular, it seems from $\delta u$ and $g_{T}$ plots that the more precise the phenomenological extractions are the larger the deviation is from lattice. But before claiming that there is a "transverse spin puzzle", more work is needed along both lines of improving the precision of phenomenological extractions and of benchmarking lattice simulations. 
Indeed, a careful determination of $\delta q$ is of paramount importance in detecting BSM effects [1].

\section{Conclusions}

In summary, we have presented a new phenomenological extraction of the transversity distribution in the framework of collinear factorization by performing for the first time a global fit of all data for the semi-inclusive production of charged pion pairs in deep-inelastic scattering, electron-positron annihilation, and proton-proton collisions. The large sensitivity of the valence down quark to the unconstrained gluon channel in di-hadron fragmentation calls for data on pion pair multiplicities, particularly in proton-proton collisions which are yet missing. The calculated (isovector) tensor charge seems compatible with most phenomenological extractions but not with lattice calculations. The effect of new COMPASS pseudodata from a future SIDIS measurement on deuterons improves the precision of the down tensor charge but confirms the general scenario.

\section{Acknowledgments}

Most of the results presented in this report have been carried out in collaboration with A. Bacchetta, to whom I am deeply indebted. This research is partially supported by the European Research Council (ERC) under the European Union's Horizon 2020 research and innovation program (Grant Agreement No. 647981, 3DSPIN).

\section{References}

[1] A. Courtoy, S. Baessler, M. Gonzalez-Alonso, and S. Liuti, Beyond-Standard-Model Tensor Interaction and Hadron Phenomenology, Phys. Rev. Lett. 115 (2015) 162001, [arXiv:1503.0681].

[2] J. C. Collins and G. A. Ladinsky, On $\pi-\pi$ correlations in polarized quark fragmentation using the linear sigma model, http://arXiv.org/abs/hep-ph/9411444.

[3] A. Bianconi, S. Boffi, R. Jakob, and M. Radici, Two-hadron interference fragmentation functions. $i$ : General framework, Phys. Rev. D62 (2000) 034008, [http://arXiv.org/abs/hep-ph/9907475].

[4] M. Radici, R. Jakob, and A. Bianconi, Accessing transversity with interference fragmentation functions, Phys. Rev. D65 (2002) 074031, [http: / / arXiv. org/abs/hep-ph/0110252].

[5] A. Bacchetta and M. Radici, Partial-wave analysis of two-hadron fragmentation functions, Phys. Rev. D67 (2003) 094002, [hep-ph/ 0212300$].$

[6] D. Boer, R. Jakob, and M. Radici, Interference fragmentation functions in electron positron annihilation, Phys. Rev. D67 (2003) 094003, [hep-ph/ 0302232 ].

[7] A. Bacchetta, F. A. Ceccopieri, A. Mukherjee, and M. Radici, Asymmetries involving dihadron fragmentation functions: from DIS to e+e-annihilation, Phys. Rev. D79 (2009) 034029, [arXiv:0812.0611].

[8] A. Courtoy, A. Bacchetta, M. Radici, and A. Bianconi, First extraction of Interference Fragmentation Functions from $e^{+} e^{-}$data, Phys.Rev. D85 (2012) 114023, [arXiv:1202.0323]. 
[9] H. H. Matevosyan, A. Bacchetta, D. Boer, A. Courtoy, A. Kotzinian, M. Radici, and A. W. Thomas, Semi-inclusive production of two back-to-back hadron pairs in $e^{+} e^{-}$annihilation revisited, Phys. Rev. D97 (2018), no. 7 074019, [arXiv: 1802 .0157].

[10] A. Bacchetta and M. Radici, Dihadron interference fragmentation functions in proton- proton collisions, Phys. Rev. D70 (2004) 094032, [hep-ph/ 0409174$].$

[11] M. Radici, A. M. Ricci, A. Bacchetta, and A. Mukherjee, Exploring universality of transversity in proton-proton collisions, Phys. Rev. D94 (2016), no. 3 034012, [arXiv: 1604 . 0658 ].

[12] HERMES Collaboration, A. Airapetian et al., Evidence for a Transverse Single-Spin Asymmetry in Leptoproduction of pi+pi-Pairs, JHEP 06 (2008) 017, [arXiv : 0803 . 2367].

[13] COMPASS Collaboration, C. Adolph et al., Transverse spin effects in hadron-pair production from semi-inclusive deep inelastic scattering, Phys.Lett. B713 (2012) 10-16, [arXiv: 1202 . 6150].

[14] COMPASS Collaboration, C. Adolph et al., A high-statistics measurement of transverse spin effects in dihadron production from muon-proton semi-inclusive deep-inelastic scattering, Phys.Lett. $\mathbf{B 7 3 6}$ (2014) 124-131, [arXiv:1401.7873].

[15] Belle Collaboration Collaboration, A. Vossen et al., Observation of transverse polarization asymmetries of charged pion pairs in e+e-annihilation near sqrt s=10.58 GeV, Phys.Rev.Lett. 107 (2011) 072004, [arXiv: 1104.2425$].$

[16] A. Bacchetta, A. Courtoy, and M. Radici, First glances at the transversity parton distribution through dihadron fragmentation functions, Phys.Rev.Lett. 107 (2011) 012001, [arXiv:1104.3855].

[17] A. Bacchetta, A. Courtoy, and M. Radici, First extraction of valence transversities in a collinear framework, JHEP 1303 (2013) 119, [arXiv: 1212. 3568].

[18] M. Radici, A. Courtoy, A. Bacchetta, and M. Guagnelli, Improved extraction of valence transversity distributions from inclusive dihadron production, JHEP 05 (2015) 123, [arXiv : 1503 . 034 9].

[19] STAR Collaboration, L. Adamczyk et al., Observation of Transverse Spin-Dependent Azimuthal Correlations of Charged Pion Pairs in $p^{\uparrow}+p$ at $\sqrt{s}=200$ GeV, Phys. Rev. Lett. 115 (2015) 242501, [arXiv:1504.0041].

[20] M. Radici and A. Bacchetta, First Extraction of Transversity from a Global Analysis of Electron-Proton and Proton-Proton Data, Phys. Rev. Lett. 120 (2018), no. 19192001, [arXiv:1802.0521].

[21] COMPASS Collaboration, F. Bradamante, The future SIDIS measurement on transversely polarized deuterons by the COMPASS Collaboration, in 23rd International Symposium on Spin Physics (SPIN 2018) Ferrara, Italy, September 10-14, 2018, 2018. arXiv: 1812.0728.

[22] Z.-B. Kang, A. Prokudin, P. Sun, and F. Yuan, Extraction of Quark Transversity Distribution and Collins Fragmentation Functions with QCD Evolution, Phys. Rev. D93 (2016), no. 1014009, [arXiv:1505.0558].

[23] M. Anselmino, M. Boglione, U. D’Alesio, S. Melis, F. Murgia, et al., Simultaneous extraction of transversity and Collins functions from new SIDIS and e+e-data, Phys.Rev. D87 (2013) 094019 , [arXiv:1303.3822].

[24] H.-W. Lin, W. Melnitchouk, A. Prokudin, N. Sato, and H. Shows, First Monte Carlo Global Analysis of Nucleon Transversity with Lattice QCD Constraints, Phys. Rev. Lett. 120 (2018), no. 15152502 , [arXiv:1710.0985]. 
[25] T. Bhattacharya, V. Cirigliano, S. Cohen, R. Gupta, H.-W. Lin, and B. Yoon, Axial, Scalar and Tensor Charges of the Nucleon from 2+1+1-flavor Lattice QCD, Phys. Rev. D94 (2016), no. 5 054508, [arXiv:1606.0704].

[26] R. Gupta, B. Yoon, T. Bhattacharya, V. Cirigliano, Y.-C. Jang, and H.-W. Lin, Flavor diagonal tensor charges of the nucleon from (2+1+1)-flavor lattice QCD, Phys. Rev. D98 (2018), no. 9091501 , [arXiv:1808.0759].

[27] C. Alexandrou et al., Nucleon scalar and tensor charges using lattice QCD simulations at the physical value of the pion mass, Phys. Rev. D95 (2017), no. 11 114514, [arXiv: 1703.0878].

[28] NPLQCD Collaboration, E. Chang, Z. Davoudi, W. Detmold, A. S. Gambhir, K. Orginos, M. J. Savage, P. E. Shanahan, M. L. Wagman, and F. Winter, Scalar, Axial, and Tensor Interactions of Light Nuclei from Lattice QCD, Phys. Rev. Lett. 120 (2018), no. 15 152002, [arXiv:1712.0322].

[29] J. R. Green, J. W. Negele, A. V. Pochinsky, S. N. Syritsyn, M. Engelhardt, and S. Krieg, Nucleon Scalar and Tensor Charges from Lattice QCD with Light Wilson Quarks, Phys. Rev. D86 (2012) 114509, [arXiv:1206.4527].

[30] G. S. Bali, S. Collins, B. Glaessle, M. Goeckeler, J. Najjar, R. H. Roedl, A. Schaefer, R. W. Schiel, W. Soeldner, and A. Sternbeck, Nucleon isovector couplings from $N_{f}=2$ lattice QCD, Phys. Rev. D91 (2015), no. 5 054501, [arXiv: 1412.7336]. 